\title{
Correction to: Rathke's cleft cysts: a 6-year experience of surgery vs. observation with comparative volumetric analysis
}

Garni Barkhoudarian $^{1}$ (1) - Sheri K. Palejwala ${ }^{1}$. Shaheryar Ansari ${ }^{1} \cdot$ Amalia A. Eisenberg $^{1} \cdot$ Xiang Huang $^{1}$. Chester F. Griffiths ${ }^{1} \cdot$ Pejman Cohan ${ }^{1} \cdot$ Sarah Rettinger ${ }^{1} \cdot$ Norman Lavin $^{1} \cdot$ Daniel F. Kelly ${ }^{1}$

Published online: 10 July 2019

(c) Springer Science+Business Media, LLC, part of Springer Nature 2019

\section{Correction to: Pituitary}

https://doi.org/10.1007/s11102-019-00962-y

The original version of this article unfortunately contained errors in legend numbers of Fig. 2 caption. Figure 2 with

Publisher's Note Springer Nature remains neutral with regard to jurisdictional claims in published maps and institutional affiliations. corrected caption is given below.
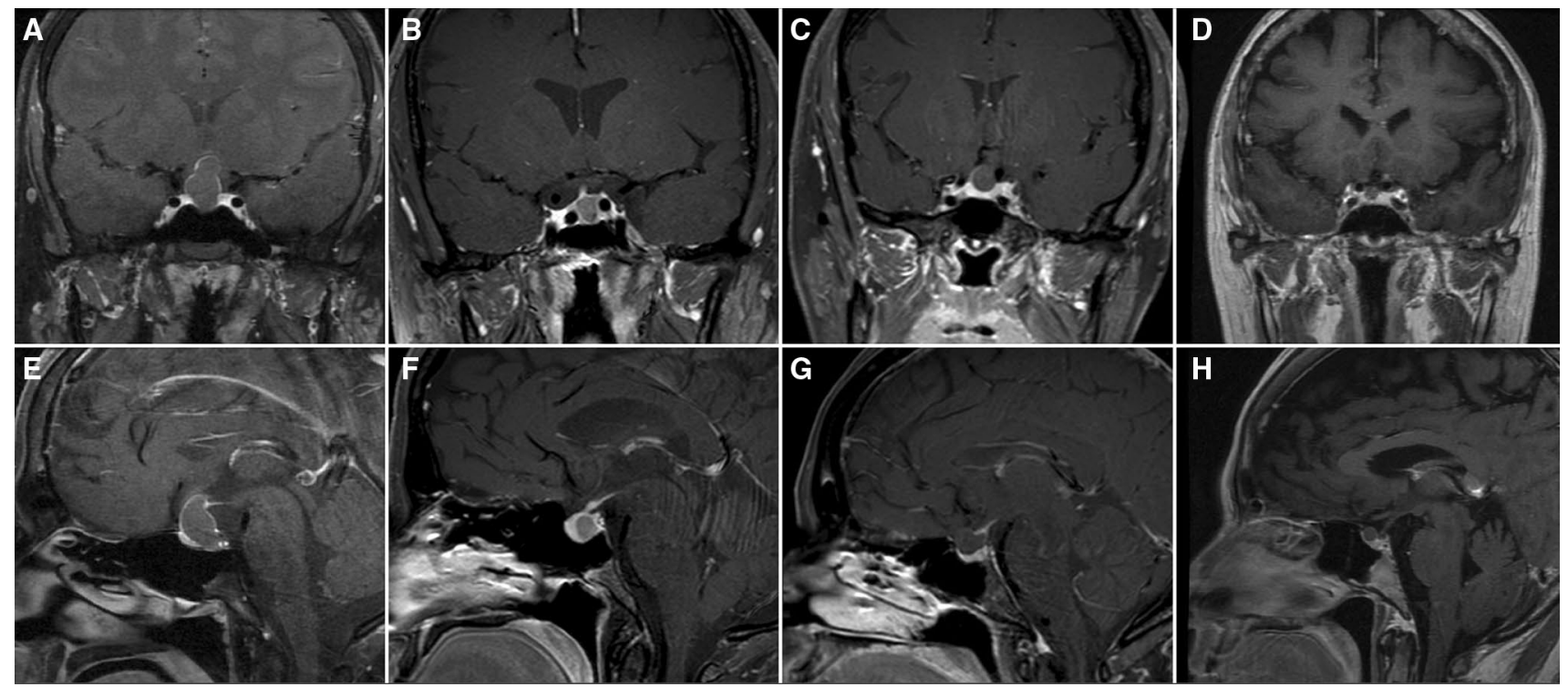

Fig. 2 Coronal and sagittal pituitary protocol MRI images of: a, e intraglandular RCC treated surgically; $\mathbf{b}$, $\mathbf{f}$ intraglandular RCC managed with observation; c, $\mathbf{g}$ supraglandular RCC treated surgically; $\mathbf{d}, \mathbf{h}$ supraglandular RCC managed with observation

The original article can be found online at https://doi.org/10.1007/ s11102-019-00962-y.

\footnotetext{
Garni Barkhoudarian

barkhoudariang@jwci.org

1 Pacific Neuroscience Institute, John Wayne Cancer Institute at Providence's Saint John's Health Center, 2125 Arizona Ave., Santa Monica, CA 90404, USA
} 